\title{
Church-Rosser property and intersection types
}

\author{
George Koletsos \\ Department of Computer Science \\ National Technical Univ. of Athens \\ GR-I5773 Zografou, Greece \\ koletsos@math.ntua.gr \\ George Stavrinos* \\ MPLA, Department of Mathematics \\ Univ. of Athens \\ GR-I5784 Zografou, Greece \\ g.stavrinos@math.ntua.gr \\ Received by Greg Restall \\ Published August 4, 2008 \\ http://www.philosophy. unimelb.edu.au/ajl/2008 \\ (C) 2008 George Koletsos and George Stavrinos
}

\begin{abstract}
We give a proof via reducibility of the Church-Rosser property for the system $D$ of $\lambda$-calculus with intersection types. As a consequence we can get the confluence property for developments directly, without making use of the strong normalization property for developments, by using only the typability in D and a suitable embedding of developments in this system. As an application we get a proof of the Church-Rosser theorem for the untyped $\lambda$-calculus.
\end{abstract}

Keywords: $\lambda$-calculus, Church-Rosser property, developments, intersection types, reducibility.

\section{INTRODUCTION}

The Church-Rosser property (or confluence property) is a central property of $\lambda$-calculus. It has known many different proofs since it was first proved by A. Church and J.B. Rosser in 1936 [3]. Some of the classical proofs are contained in [2]. The property was also proved for the simply typed $\lambda$-calculus $\lambda_{\rightarrow}$ by G. Koletsos [IO] and R. Statman [I5] using the reducibility method and logical relations, respectively.

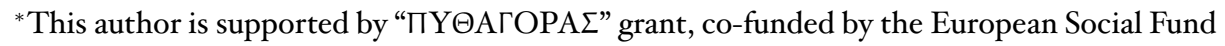
(75\%) and the Hellenic Ministry of Education (25\%) under Operational Programme on Education and Initial Vocational Training (EПEAEK II). 
In this paper we prove, using a reducibility argument, the Church-Rosser property for the system D of $\lambda$-calculus with intersection types. The intersection types assignment systems were introduced by M. Coppo, M. DezaniCiancaglini, and B. Venneri for characterizing via typability fundamental properties of the untyped $\lambda$-calculus such as solvability and strong normalization [4, 5, 6. 6 . The system D is treated extensively by J.-L. Krivine in [13] where characterizations of normalization properties are given via the reducibility method by interpreting the types with suitable sets of $\lambda$-terms. A detailed study of this method for proving general properties of $\lambda$-calculus can be found in [7, 8 , [II], and [9]. In our proof of the Church-Rosser property for system D we adapt the reducibility method of [II] to this system.

As a consequence of the Church-Rosser property for system $D$ we get the confluence of a special kind of reduction called development. A development is a restricted reduction in which we select some initial redexes and keep reducing only them and their residuals throughout the reduction. In this way all developments are finite and have unique normal form [2, Chapter II]. This property of developments was originally used by A. Church to prove the Church-Rosser property for the untyped $\lambda$-calculus. In [2] the confluence of developments is proved by using the well-known Newman's lemma, i.e. strong normalization and the weak Church-Rosser property imply the Church-Rosser property $[14]$, so the strong normalization of developments is used as a prerequisite. In our proof, the confluence of developments comes directly, without using the strong normalization property, from the Church-Rosser property for system $\mathrm{D}$ and by embedding the untyped $\lambda$-calculus into the system $D$. Note though that strong normalization has equal strength as typability in D (see [I3], page 65] and [I, Theorem 7.4.II]).

As an application we can easily get a proof of the Church-Rosser theorem for the full untyped $\lambda$-calculus.

In section 2 of the paper we introduce the basic notions and prove via reducibility the Church-Rosser property for system D. In section 3 we define precisely an operator that establishes the embedding of the untyped $\lambda$-calculus into D and prove the confluence of developments. Finally, in section 4 we use the previous result to prove the Church-Rosser theorem for the untyped $\lambda$-calculus.

\section{THE CHURCH-ROSSER PROPERTY FOR SYSTEM D}

We start this section by presenting briefly some well-known definitions from $\lambda$-calculus and system $D$. The notation, terminology and the syntactic conventions are adopted mainly from [13]. 


\section{PRELIMINARY DEFINITIONS}

The types of $\mathrm{D}$ are the "propositional sentences" built inductively from the variables $X, Y, \ldots$ (the type variables) and the connectives $\cap$ and $\rightarrow$. We use capital letters $X, Y, \ldots$ for the type variables and small letters $x, y, \ldots$ for the individual variables by which we construct the $\lambda$-terms.

The untyped $\lambda$-terms are built inductively starting from the variables $x, y, \ldots$ and using the following rule: if $t$ and $u$ are terms then $(t) u(a p p l i c a t i o n)$ and $\lambda x . t\left(\lambda\right.$-abstraction) are terms. For simplicity we write $(u) t_{1} t_{2} \ldots t_{n}$ or even $u t_{1} t_{2} \ldots t_{n}$ for $\left(\ldots\left((u) t_{1}\right) t_{2} \ldots\right) t_{n}$. The $\lambda x$ of a $\lambda$-abstraction term acts here as a variable binder and so we must distinguish between the bound and free occurrences of a variable in a term. We denote by $\mathrm{FV}(\mathrm{u})$ the set of free variables in the term $u$ and we write $u\left[t_{1} / x_{1}, \ldots, t_{n} / x_{n}\right]$ for the "simultaneous" substitution of the free occurrences of $x_{1}, \ldots, x_{n}$ in $u$ by $t_{1}, \ldots, t_{n}$, respectively. When necessary we also adopt Barendregt's variable convention so that all bound variables are chosen to be different from the free variables.

A context $\Gamma$ is a finite set of declarations $x: A$ where $x$ is an individual variable, $A$ is a type, and no $x$ appears twice. $x$ : $A$ means "variable $x$ has type $A$ ". We write $\Gamma, x: A$ for the context $\Gamma \cup\{x: A\}$ where we always assume that $x$ does not appear in $\Gamma$.

We define inductively the notion "in context $\Gamma$, term $t$ has type $A$ " written $\Gamma \vdash_{\mathrm{D}} \mathrm{t}: \mathrm{A}$ (or more simply $\left.\Gamma \vdash t: A\right)$ :

Rule I. Г, $\mathrm{x}: \mathrm{A} \vdash \mathrm{x}: \mathrm{A}$ (hypothesis)

Rule 2. $\frac{\Gamma, x: A \vdash t: B}{\Gamma \vdash \lambda x \cdot t: A \rightarrow B}(\rightarrow-$ introduction $)$

Rule 3. $\frac{\Gamma \vdash \mathrm{t}: \mathrm{A} \quad \Gamma \vdash \mathrm{u}: \mathrm{A} \rightarrow \mathrm{B}}{\Gamma \vdash(\mathrm{u}) \mathrm{t}: \mathrm{B}}(\rightarrow$-elimination $)$

Rule 4. $\frac{\Gamma \vdash t: A \cap B}{\Gamma \vdash t: A}\left(\cap_{1}\right.$-elimination $) \frac{\Gamma \vdash t: A \cap B}{\Gamma \vdash t: B}\left(\cap_{2}\right.$-elimination $)$

Rule 5. $\frac{\Gamma \vdash \mathrm{t}: \mathrm{A} \quad \Gamma \vdash \mathrm{t}: \mathrm{B}}{\Gamma \vdash \mathrm{t}: \mathrm{A} \cap \mathrm{B}}(\cap$-introduction $)$

Note that $\cap$ is a special conjunction which behaves rather as a set-theoretic intersection.

We call $\Gamma \vdash t: A$ a typing of $t$. If a term gets a typing by the above rules then it is a typed or typable term. It is easy to check that $\mathrm{FV}(\mathrm{t}) \subseteq\left\{\mathrm{x}_{1}, \ldots, \mathrm{x}_{\mathrm{k}}\right\}$ whenever $x_{1}: A_{1}, \ldots, x_{k}: A_{k} \vdash t: A$.

Let $\Lambda$ denote the set of all (untyped) $\lambda$-terms. If $X$ and $y$ are subsets of $\Lambda$, we define $X \rightarrow Y$ by:

$$
\mathrm{u} \in(X \rightarrow \mathrm{y}) \stackrel{\text { def }}{\Longleftrightarrow} \forall \mathrm{t} \in X,(\mathrm{u}) \mathrm{t} \in \mathrm{y}
$$


The only reduction rule considered is $\beta$-reduction (notation $\stackrel{*}{\longrightarrow} \beta$ ) defined as the contextual, reflexive and transitive closure of the relation

$$
(\lambda x . u) v \longrightarrow \beta u[v / x]
$$

between a redex $(\lambda x . u) v$ and its contractum $u[v / x]$. We write $t \longrightarrow \beta t^{\prime}$ when $t^{\prime}$ is obtained from $t$ by contracting one redex in $t$, and $t \stackrel{*}{\longrightarrow}_{\beta} t^{\prime}$ when $t^{\prime}$ is obtained by a finite sequence (possibly empty) of contractions from $t$.

We say that a term $t$ has the Church-Rosser property (t has CR) wrt $\stackrel{*}{\longrightarrow}_{\beta}$, if there exists a term $t_{3}$ such that $t_{1} \stackrel{*}{\longrightarrow} \beta t_{3}$ and $t_{2} \stackrel{*}{\longrightarrow} \beta t_{3}$ whenever $t \stackrel{*}{\longrightarrow} \beta t_{1}$ and $t \stackrel{*}{\longrightarrow} \beta t_{2}$. The $\beta$-reduction relation (or any other reduction relation defined on $\lambda$-terms) has the Church-Rosser property or is confluent if every term has the Church-Rosser property wrt $\stackrel{*}{\longrightarrow} \beta$ (wrt that relation, respectively).

We define next the formal machinery that will be needed in our work.

DEFINITION I A direct reduct of an application term $u v_{1} \ldots v_{n}(n \geqslant 1)$ is a term $u^{\prime} v_{1}^{\prime} \ldots v_{n}^{\prime}$ such that $u \stackrel{*}{\longrightarrow} \beta u^{\prime}, v_{1} \stackrel{*}{\longrightarrow}_{\beta} v_{1}^{\prime}, \ldots, v_{n} \stackrel{*}{\longrightarrow}_{\beta} v_{n}^{\prime}$ (therefore $\left.u v_{1} \ldots v_{n} \stackrel{*}{\longrightarrow} \beta u^{\prime} v_{1}^{\prime} \ldots v_{n}^{\prime}\right)$.

We must note that a direct reduct of $u v_{1} \ldots v_{n}$ is defined wrt a fixed number $n$ of operands, i.e. wrt a specific presentation of $u v_{1} \ldots v_{n}$ considered as a term constructed from $u$ by the $n$ consecutive applications $(u) v_{1},\left(u v_{1}\right) v_{2}, \ldots$, $\left(u v_{1} \ldots v_{n-1}\right) v_{n}$. So any direct reduct of $u v_{1} \ldots v_{n}$ invariantly has the same form of presentation and a direct reduct of a direct reduct of $u v_{1} \ldots v_{n}$ is always a direct reduct of $u v_{1} \ldots v_{n}$.

LEMMA 2 If $u v_{1} \ldots v_{n} \stackrel{*}{\longrightarrow} \beta w$ and $w$ is not a direct reduct of $u v_{1} \ldots v_{n}$ then there exists a direct reduct $u^{\prime} v_{1}^{\prime} \ldots v_{n}^{\prime}$ of $u v_{1} \ldots v_{n}$ where $u^{\prime}=\lambda x . u^{\prime \prime}$ for some term $u^{\prime \prime}$, and $\mathrm{u}^{\prime \prime}\left[v_{1}^{\prime} / \mathrm{x}\right] v_{2}^{\prime} \ldots v_{\mathrm{n}}^{\prime} \stackrel{*}{\longrightarrow} \beta$ w, i.e.

$$
u v_{1} \ldots v_{n} \stackrel{*}{\longrightarrow} \beta\left(\lambda x . u^{\prime \prime}\right) v_{1}^{\prime} \ldots v_{n}^{\prime} \longrightarrow_{\beta} u^{\prime \prime}\left[v_{1}^{\prime} / x\right] v_{2}^{\prime} \ldots v_{n}^{\prime} \stackrel{*}{\longrightarrow} \beta w
$$

Proof: Let $w^{\prime}$ be the first non direct reduct of $u v_{1} \ldots v_{n}$ in the reduction $u v_{1} \ldots v_{n} \stackrel{*}{\longrightarrow} \beta w$. Then

$$
u v_{1} \ldots v_{n} \stackrel{*}{\longrightarrow} \beta u^{\prime} v_{1}^{\prime} \ldots v_{n}^{\prime} \longrightarrow_{\beta} w^{\prime} \stackrel{*}{\longrightarrow}_{\beta} w
$$

where $u^{\prime} v_{1}^{\prime} \ldots v_{n}^{\prime}$ is a direct reduct of $u v_{1} \ldots v_{n}$. So $w^{\prime}$ cannot be a direct reduct of $u^{\prime} v_{1}^{\prime} \ldots v_{n}^{\prime}$ because any direct reduct of $u^{\prime} v_{1}^{\prime} \ldots v_{n}^{\prime}$ is obviously a direct reduct of $u v_{1} \ldots v_{n}$. The only way to get a non direct reduct of $u^{\prime} v_{1}^{\prime} \ldots v_{n}^{\prime}$ is by contracting a redex which is not inside in any of $u^{\prime}, v_{1}^{\prime}, \ldots, v_{n}^{\prime}$ and this is possible only if $u^{\prime}$ is a $\lambda$-abstraction $\lambda x \cdot u^{\prime \prime}$ and the redex contracted is $\left(\lambda x . u^{\prime \prime}\right) v_{1}^{\prime}$. Then $w^{\prime}=u^{\prime \prime}\left[v_{1}^{\prime} / x\right] v_{2}^{\prime} \ldots v_{n}^{\prime}$.

LEMMa 3 If $\mathrm{t} \stackrel{*}{\longrightarrow} \beta \mathrm{t}^{\prime}$ and $\mathrm{u} \stackrel{*}{\longrightarrow} \beta \mathrm{u}^{\prime}$ then $\mathrm{u}[\mathrm{t} / \mathrm{x}] \stackrel{*}{\longrightarrow} \beta \mathrm{u}^{\prime}\left[\mathrm{t}^{\prime} / \mathrm{x}\right]$.

Proof: The proof can be found in any textbook on $\lambda$-calculus, for example [2, page 55 .

\footnotetext{
${ }^{\mathrm{I}}$ with respect to
} 
DEFinition 4 We define $\mathcal{C R}$ to be the set of $\lambda$-terms that have the ChurchRosser property, i.e. $\mathcal{C R} \stackrel{\text { def }}{=}\{\mathrm{t} \in \Lambda: \mathrm{t}$ has $\mathrm{CR}\}$, and $\mathcal{C} \mathcal{R}_{0}$ the set of $\lambda$-terms of the form $x v_{1} \ldots v_{n}(n \geqslant 0)$ where $x$ is a variable and $v_{1}, \ldots, v_{n} \in \mathcal{C} \mathcal{R}$.

DEFINITION $5 X \Lambda$ is said to be saturated when for all terms $u, t, t_{1}, \ldots, t_{n}$ $(n \geqslant 0)$ and for every variable $x$ we have:

$$
u[t / x] t_{1} \ldots t_{n} \in X \Rightarrow(\lambda x . u) t t_{1} \ldots t_{n} \in X
$$

LEMMA 6 (I) If $X, y \subseteq \wedge$ are saturated then $X \cap y$ is saturated.

(2) If $y \subseteq \Lambda$ is saturated and $X \subseteq 1$ then $X \rightarrow y$ is saturated.

Proof: (I) If $u[t / x] t_{1} \ldots t_{n} \in X \cap y$ then $(\lambda x . u) t t_{1} \ldots t_{n} \in X, y$.

(2) If $u[t / x] t_{1} \ldots t_{n} \in X \rightarrow y$ then $u[t / x] t_{1} \ldots t_{n} t_{0} \in y$ for every $t_{0} \in X$, and because $y$ is saturated $(\lambda x . u) t t_{1} \ldots t_{n} t_{0} \in Y$. So $(\lambda x . u) t t_{1} \ldots t_{n} \in X \rightarrow Y$.

PROPOSITION 7 CR is saturated.

Proof: Suppose that $u[t / x] t_{1} t_{2} \ldots t_{n} \in \mathcal{E R}$. To prove that $(\lambda x . u) t t_{1} \ldots t_{n} \in \mathcal{E} \mathcal{R}$ we suppose that $(\lambda x . u) t t_{1} \ldots t_{n} \stackrel{*}{\longrightarrow} \beta v$ and $(\lambda x . u) t t_{1} \ldots t_{n} \stackrel{*}{\longrightarrow}_{\beta} w$ [Figure I].

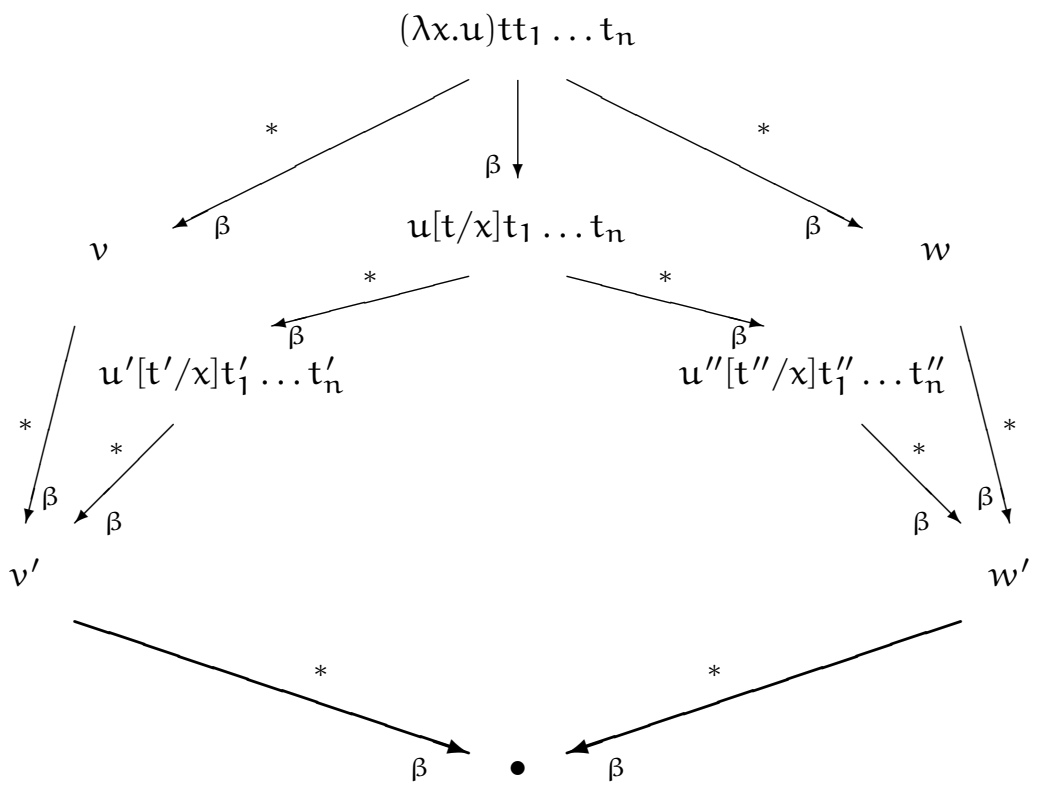

Figure I: Diagram of reductions for the proof of Proposition 7

Consider the left reduction $(\lambda x . u) t t_{1} \ldots t_{n} \stackrel{*}{\longrightarrow} \beta v$. Then either $v$ is a direct reduct of $(\lambda x . u) t t_{1} \ldots t_{n}$ or it is not.

In the first case, there exist terms $u^{\prime}, t^{\prime}, t_{1}^{\prime}, \ldots, t_{n}^{\prime}$ such that

$$
u \stackrel{*}{\longrightarrow} \beta u^{\prime}, \quad t \stackrel{*}{\longrightarrow}_{\beta} t^{\prime}, t_{1} \stackrel{*}{\longrightarrow}_{\beta} t_{1}^{\prime}, \ldots, t_{n} \stackrel{*}{\longrightarrow}_{\beta} t_{n}^{\prime},
$$


and $(\lambda x . u) t t_{1} \ldots t_{n} \stackrel{*}{\longrightarrow} \beta\left(\lambda x \cdot u^{\prime}\right) t^{\prime} t_{1}^{\prime} \ldots t_{n}^{\prime}=v$. Then $v \longrightarrow_{\beta} u^{\prime}\left[t^{\prime} / x\right] t_{1}^{\prime} \ldots t_{n}^{\prime}$ (by one contraction). Let $v^{\prime}=u^{\prime}\left[t^{\prime} / x\right] t_{1}^{\prime} \ldots t_{n}^{\prime}$. By Lemma 3, $u[t / x] t_{1} t_{2} \ldots t_{n} \stackrel{*}{\longrightarrow} \beta v^{\prime}$ and so $v$ and $u[t / x] t_{1} t_{2} \ldots t_{n}$ both reduce to $v^{\prime}$.

In the second case, by Lemma 2 there exists a direct reduct $\left(\lambda x \cdot u^{\prime}\right) t^{\prime} t_{1}^{\prime} \ldots t_{n}^{\prime}$ of $(\lambda x . u) t t_{1} \ldots t_{n}$ such that

$$
u \stackrel{*}{\longrightarrow} \beta u^{\prime}, \quad t \stackrel{*}{\longrightarrow} \beta t^{\prime}, \quad t_{1} \stackrel{*}{\longrightarrow} \beta t_{1}^{\prime}, \ldots, t_{n} \stackrel{*}{\longrightarrow} \beta t_{n}^{\prime},
$$

and

$$
(\lambda x . u) t t_{1} \ldots t_{n} \stackrel{*}{\longrightarrow}_{\beta}\left(\lambda x \cdot u^{\prime}\right) t^{\prime} t_{1}^{\prime} \ldots t_{n}^{\prime} \longrightarrow_{\beta} u^{\prime}\left[t^{\prime} / x\right] t_{1}^{\prime} \ldots t_{n}^{\prime} \stackrel{*}{\longrightarrow}_{\beta} v
$$

Let $v^{\prime}=v$. By Lemma $3, u[t / x] t_{1} t_{2} \ldots t_{n} \stackrel{*}{\longrightarrow} \beta u^{\prime}\left[t^{\prime} / x\right] t_{1}^{\prime} \ldots t_{n}^{\prime}$ and so $u[t / x] t_{1} t_{2} \ldots t_{n} \stackrel{*}{\longrightarrow} \beta v^{\prime}$.

In both cases there exists a term $v^{\prime}$ such that $\nu \stackrel{*}{\longrightarrow} \nu^{\prime}$ (by one or zero contractions) and

$$
\mathrm{u}[\mathrm{t} / \mathrm{x}] \mathrm{t}_{1} \ldots \mathrm{t}_{\mathrm{n}} \stackrel{*}{\longrightarrow} \beta v^{\prime}
$$

As the same argument holds also for the right reduction $(\lambda x . u) t t_{1} \ldots t_{n} \stackrel{*}{\longrightarrow} \beta w$, we can also obtain a term $w^{\prime}$ such that $w \stackrel{*}{\longrightarrow} \beta w^{\prime}$ (by one or zero contractions) and

$$
\mathrm{u}[\mathrm{t} / \mathrm{x}] \mathrm{t}_{1} \ldots \mathrm{t}_{\mathrm{n}} \stackrel{*}{\longrightarrow} \beta w^{\prime}
$$

The result follows from (I), 2], and the assumption that $u[t / x] t_{1} t_{2} \ldots t_{n}$ has CR.

DEFINITION 8 An interpretation $\mathcal{J}$ is a mapping from type variables $X$ to saturated subsets of $\Lambda$, denoted by $\llbracket X \rrbracket_{\mathcal{J}}$. We inductively extend $\llbracket_{-} \rrbracket_{\mathcal{J}}$ in to a mapping from types in the system $D$ to subsets of $\Lambda$, in the following way:

(I) if $A$ is a type variable then $\llbracket A \rrbracket_{J}$ is already defined;

(2) if $A=B \cap C$ then $\llbracket A \rrbracket_{\mathcal{J}} \stackrel{\text { def }}{=} \llbracket B \rrbracket_{\mathcal{J}} \cap \llbracket C \rrbracket_{\mathcal{J}}$;

(3) if $\mathrm{A}=\mathrm{B} \rightarrow \mathrm{C}$ then $\llbracket \mathrm{A} \rrbracket_{\mathcal{J}} \stackrel{\text { def }}{=}\left(\llbracket \mathrm{B} \rrbracket_{\mathcal{J}} \rightarrow \llbracket C \rrbracket_{\mathcal{J}}\right) \bigcap \mathcal{C R}$.

REMARK 9 We interpret the types of D by suitable sets of $\lambda$-terms that will guarantee the desired Church-Rosser property, i.e. $\llbracket A \rrbracket_{\mathcal{J}} \subseteq \mathcal{C} \mathcal{R}[$ Lemma I4]. But in our variant of the reducibility method, we selected the interpretation of $\mathrm{B} \rightarrow \mathrm{C}$ to reside within $\mathcal{C R}$ in contrast to the usual reducibility interpretations where $\mathrm{B} \rightarrow \mathrm{C}$ is interpreted by $\llbracket \mathrm{B} \rrbracket_{\mathcal{J}} \rightarrow \llbracket C \rrbracket_{\mathcal{J}}$. In the latter case we would be stuck with the proof of $\mathcal{C R} \rightarrow \mathcal{C R} \subseteq \mathcal{C} \mathcal{R}$ as explained by the following reasoning.

Suppose that $t \in \mathcal{C R} \rightarrow \mathcal{C R}$ and let $t \stackrel{*}{\longrightarrow} t_{1}, t \stackrel{*}{\longrightarrow}_{\beta} t_{2}$. Then for any variable $x$ not free in $t,(t) x \stackrel{*}{\longrightarrow}_{\beta}\left(t_{1}\right) x,(t) x \stackrel{*}{\longrightarrow}_{\beta}\left(t_{2}\right) x$ and because $x \in \mathcal{C R}$ we have that $(t) x \in \mathcal{C} \mathcal{R}$. So we can find a term $u$ such that $\left(t_{1}\right) x \stackrel{*}{\longrightarrow} \beta u$ 
and $\left(t_{2}\right) x \stackrel{*}{\longrightarrow} \beta u$. If $u$ is a direct reduct of $\left(t_{1}\right) x,\left(t_{2}\right) x$ then $u=\left(t_{3}\right) x$ and $t_{1} \stackrel{*}{\longrightarrow} \beta t_{3}, t_{2} \stackrel{*}{\longrightarrow} \beta t_{3}$ therefore $t \in \mathcal{C R}$, i.e. the confluence from $t$ simulates the confluence from $(t) x$ to $u$. Otherwise, $t_{1} \stackrel{*}{\longrightarrow}_{\beta} u_{1}$ and $t_{2} \stackrel{*}{\longrightarrow} \beta u_{2}$ where $\lambda x . u \stackrel{*}{\longrightarrow} \eta u_{1}$ and $\lambda x . u \stackrel{*}{\longrightarrow} \eta u_{2}$, so $u_{1}, u_{2}$ are $\eta$-equivalent ${ }^{2}$ and the confluence from $(t) x$ to $u$ cannot be simulated by $t$ I6).

PROPOSITION IO For every interpretation $\mathcal{J}$ and every type $A, \llbracket A \rrbracket_{\mathcal{J}}$ is saturated.

Proof: By induction on the construction of type $A$. We consider only the case $\mathrm{A}=\mathrm{B} \rightarrow \mathrm{C}$.

By the $\mathrm{IH}^{3}, \llbracket C \rrbracket_{\mathcal{J}}$ is saturated. So $\llbracket \mathrm{B} \rrbracket_{\mathcal{J}} \rightarrow \llbracket C \rrbracket_{\mathcal{J}}$ is saturated [Lemma 6 ]. By Proposition 7, $\mathcal{C R}$ is saturated and therefore $\left(\llbracket \mathrm{B} \rrbracket_{\mathcal{J}} \rightarrow \llbracket C \rrbracket_{\mathcal{J}}\right) \cap \mathcal{C R}$ is saturated [Lemma 6].

THEOREM II (soundness, adequacy) Let $\mathcal{J}$ be an interpretation such that $\mathcal{e} \mathcal{R}_{0} \subseteq \llbracket \mathrm{B} \rrbracket_{\mathcal{J}} \subseteq$ CR for every type $\mathrm{B}$. If $\mathrm{x}_{1}: \mathrm{A}_{1}, \ldots, \mathrm{x}_{\mathrm{k}}: \mathrm{A}_{\mathrm{k}} \vdash \mathrm{u}: \mathrm{A}$ is a typing of $\mathrm{u}$, then for all terms $\mathrm{t}_{1} \in \llbracket A_{1} \rrbracket_{\mathcal{J}}, \ldots, \mathrm{t}_{k} \in \llbracket A_{k} \rrbracket_{\mathcal{J}}$ we have $u\left[\mathrm{t}_{1} / \mathrm{x}_{1}, \ldots, \mathrm{t}_{\mathrm{k}} / \mathrm{x}_{\mathrm{k}}\right] \in \llbracket A \rrbracket_{\mathfrak{J}}$.

Proof: We use induction on the typing in D of the term $\mathrm{u}$. Consider the last rule used:

(I) For Rule $I, u$ is one variable between $x_{1}, \ldots, x_{k}$, say $x_{i}$, and $A=A_{i}$. Then $u\left[t_{1} / x_{1}, \ldots, t_{k} / x_{k}\right]=t_{i}$ where $t_{i} \in \llbracket A_{i} \rrbracket_{J}$ by hypothesis.

(2) For Rule 2, $u=\lambda x \cdot v, A=B \rightarrow C$ and we have:

$$
\frac{x: B, x_{1}: A_{1}, \ldots, x_{k}: A_{k} \vdash v: C}{x_{1}: A_{1}, \ldots, x_{k}: A_{k} \vdash \lambda x \cdot v: B \rightarrow C}
$$

Because $x$ is a bound variable in $u$, by the variable convention we can choose $x$ such that $x \notin F V\left(t_{1} t_{2} \ldots t_{k}\right) \cup\left\{x_{1}, \ldots, x_{k}\right\}$. We want to prove that

$$
(\lambda x . v)\left[t_{1} / x_{1}, \ldots, t_{k} / x_{k}\right] \in\left(\llbracket B \rrbracket_{\mathcal{J}} \rightarrow \llbracket C \rrbracket_{\mathcal{J}}\right) \bigcap e \mathcal{R}
$$

By IH we have that (for all $t_{i} \in \llbracket A_{i} \rrbracket_{\mathcal{J}}$ )

$$
\forall \mathrm{t} \in \llbracket \mathrm{B} \rrbracket_{\mathcal{J}}, v\left[\mathrm{t} / \mathrm{x}, \mathrm{t}_{1} / \mathrm{x}_{1}, \ldots, \mathrm{t}_{\mathrm{k}} / \mathrm{x}_{\mathrm{k}}\right] \in \llbracket \mathrm{C} \rrbracket_{\mathcal{J}}
$$

(i) Because of the choice of $x$, the term $v\left[t / x, t_{1} / x_{1}, \ldots, t_{k} / x_{k}\right]$ is identical to the term $\left(v\left[t_{1} / x_{1}, \ldots, t_{k} / x_{k}\right]\right)[t / x]$ modulo renaming of bound variables. Thus

$$
\left(\lambda x . v\left[t_{1} / x_{1}, \ldots, t_{k} / x_{k}\right]\right) t \in \llbracket C \rrbracket_{\mathcal{J}}
$$

${ }^{2}$ The $\eta$-reduction relation (notation $\stackrel{*}{\longrightarrow}$ ) is defined as the contextual, reflexive and transitive closure of the the relation $\lambda x .(v) x \longrightarrow_{\eta} v$ where $x \notin \mathrm{FV}(v)$. The equivalence relation induced by $\stackrel{*}{\longrightarrow}$ is called $\eta$-equivalence.

3 induction hypothesis 
because $\llbracket C \rrbracket_{\mathfrak{J}}$ is saturated by Proposition $\mathrm{IO}$, So

$$
\lambda x . v\left[t_{1} / x_{1}, \ldots, t_{k} / x_{k}\right] \in \llbracket B \rrbracket_{\mathcal{J}} \rightarrow \llbracket C \rrbracket_{\mathcal{J}}
$$

By the choice of $x$,

$$
\lambda x . v\left[t_{1} / x_{1}, \ldots, t_{k} / x_{k}\right]=(\lambda x . v)\left[t_{1} / x_{1}, \ldots, t_{k} / x_{k}\right]
$$

so

$$
(\lambda x . v)\left[t_{1} / x_{1}, \ldots, t_{k} / x_{k}\right] \in \llbracket B \rrbracket_{\mathcal{J}} \rightarrow \llbracket C \rrbracket_{\mathcal{J}}
$$

(ii) By hypothesis $\mathcal{C} \mathcal{R}_{0} \subseteq \llbracket \mathrm{B} \rrbracket_{\mathcal{J}}, \llbracket C \rrbracket_{\mathcal{J}} \subseteq \mathcal{C} \mathcal{R}$ and because $x \in \mathcal{C} \mathcal{R}_{0}$ we have that

$$
v\left[x / x, t_{1} / x_{1}, \ldots, t_{k} / x_{k}\right] \in \mathcal{C} \mathcal{R}
$$

Thus

$$
v\left[t_{1} / x_{1}, \ldots, t_{k} / x_{k}\right] \in \mathcal{C R}
$$

Since abstraction on the outside of a term does not add redexes $\lambda x . v\left[t_{1} / x_{1}, \ldots, t_{k} / x_{k}\right]$ has CR and because of the choice of $x$,

$$
\lambda x . v\left[t_{1} / x_{1}, \ldots, t_{k} / x_{k}\right]=(\lambda x . v)\left[t_{1} / x_{1}, \ldots, t_{k} / x_{k}\right]
$$

So

$$
(\lambda x . v)\left[t_{1} / x_{1}, \ldots, t_{k} / x_{k}\right] \in \mathcal{C R}
$$

(3) For Rule 3, $u=w v$ and for some type B we have:

$$
\frac{x_{1}: A_{1}, \ldots, x_{k}: A_{k} \vdash v: B \quad x_{1}: A_{1}, \ldots, x_{k}: A_{k} \vdash w: B \rightarrow A}{x_{1}: A_{1}, \ldots, x_{k}: A_{k} \vdash w v: A}
$$

By IH, $v\left[\mathrm{t}_{1} / \mathrm{x}_{1}, \ldots, \mathrm{t}_{\mathrm{k}} / \mathrm{x}_{\mathrm{k}}\right] \in \llbracket \mathrm{B} \rrbracket_{\mathcal{J}}$ and

$$
w\left[\mathrm{t}_{1} / \mathrm{x}_{1}, \ldots, \mathrm{t}_{\mathrm{k}} / \mathrm{x}_{\mathrm{k}}\right] \in\left(\llbracket \mathrm{B} \rrbracket_{\mathcal{J}} \rightarrow \llbracket A \rrbracket_{\mathcal{J}}\right) \bigcap \mathcal{C R}
$$

so

$$
\left(w\left[t_{1} / x_{1}, \ldots, t_{k} / x_{k}\right]\right) v\left[t_{1} / x_{1}, \ldots, t_{k} / x_{k}\right] \in \llbracket A \rrbracket_{J}
$$

i.e. $(w v)\left[t_{1} / x_{1}, \ldots, t_{k} / x_{k}\right] \in \llbracket A \rrbracket_{J}$.

(4) For Rule 4, we have for some type B:

$$
\frac{x_{1}: A_{1}, \ldots, x_{k}: A_{k} \vdash u: A \cap B}{x_{1}: A_{1}, \ldots, x_{k}: A_{k} \vdash u: A}
$$

By IH, $u\left[t_{1} / x_{1}, \ldots, t_{k} / x_{k}\right] \in \llbracket A \rrbracket_{\mathcal{J}} \cap \llbracket B \rrbracket_{\mathcal{J}}$, so $u\left[t_{1} / x_{1}, \ldots, t_{k} / x_{k}\right] \in \llbracket A \rrbracket_{\mathcal{J}}$ and the same holds for $\cap_{2}$-elimination. 
(5) For Rule 5, $A=B \cap C$ and we have:

$$
\frac{x_{1}: A_{1}, \ldots, x_{k}: A_{k} \vdash u: B \quad x_{1}: A_{1}, \ldots, x_{k}: A_{k} \vdash u: C}{x_{1}: A_{1}, \ldots, x_{k}: A_{k} \vdash u: B \cap C}
$$

By IH, $u\left[t_{1} / x_{1}, \ldots, t_{k} / x_{k}\right] \in \llbracket B \rrbracket_{\mathcal{J}}$ and $u\left[t_{1} / x_{1}, \ldots, t_{k} / x_{k}\right] \in \llbracket C \rrbracket_{\mathcal{J}}$, so $u\left[t_{1} / x_{1}, \ldots, t_{k} / x_{k}\right] \in \llbracket B \cap C \rrbracket_{\mathfrak{J}}$.

LEMMA I 2 $\mathcal{L} \mathcal{R}_{0} \subseteq \mathcal{e} \mathcal{R}$

Proof: Suppose that $x v_{1} \ldots v_{n} \stackrel{*}{\longrightarrow} \beta u$ and $x v_{1} \ldots v_{n} \stackrel{*}{\longrightarrow} \beta w$ where $v_{1}, \ldots, v_{n}$ have CR. Then $u$ and $w$ must necessarily be direct reducts of $x v_{1} \ldots v_{n}$ [Lemma 2] of the form $u=x v_{1}^{\prime} \ldots v_{n}^{\prime}, w=x v_{1}^{\prime \prime} \ldots v_{n}^{\prime \prime}$ and $v_{i} \stackrel{*}{\longrightarrow}_{\beta} v_{i}^{\prime}, v_{i} \stackrel{*}{\longrightarrow}_{\beta} v_{i}^{\prime \prime}$ for all $i \in\{1, \ldots, n\}$. But then there exist terms $v_{i}^{\prime \prime \prime}(1 \leqslant i \leqslant n)$ such that $v_{i}^{\prime} \stackrel{*}{\longrightarrow}_{\beta} v_{i}^{\prime \prime \prime}$ and $v_{i}^{\prime \prime} \stackrel{*}{\longrightarrow}_{\beta} v_{i}^{\prime \prime \prime}$. By using the properties of $\beta$-reduction we can conclude that $u \stackrel{*}{\longrightarrow} \beta \times v_{1}^{\prime \prime \prime} \ldots v_{n}^{\prime \prime \prime}$ and $w \stackrel{*^{*}}{\longrightarrow} \times v_{1}^{\prime \prime \prime} \ldots v_{n}^{\prime \prime \prime}$.

LEMMA I3 $2 \mathcal{R}_{0} \subseteq\left(\mathcal{C R} \rightarrow \mathrm{eR}_{0}\right)$

Proof: Suppose that $x v_{1} \ldots v_{n} \in \mathcal{e} \mathcal{R}_{0}$ and $v \in \mathcal{C} \mathcal{R}$. Then by definition of $\mathcal{e} \mathcal{R}_{0}$, $v_{i} \in \mathcal{C} \mathcal{R}(1 \leqslant i \leqslant n)$ and therefore $x v_{1} \ldots v_{n} v \in \mathcal{C} \mathcal{R}_{0}$.

LEMMA I4 If $\mathcal{J}$ is an interpretation such that $\mathcal{C} \mathcal{R}_{0} \subseteq \llbracket X \rrbracket_{\mathcal{J}} \subseteq$ eR for every type variable $\mathrm{X}$, then $\mathrm{CR}_{0} \subseteq \llbracket A \rrbracket_{\mathcal{J}} \subseteq \mathcal{C} \mathcal{R}$ for every type $\mathrm{A}$.

Proof: We use induction on the construction of type A.

(I) If $A$ is a type variable $X$ then the result follows from the assumption.

(2) If $A=B \cap C$ then by $\mathrm{IH}, e \mathcal{R}_{0} \subseteq \llbracket \mathrm{B} \rrbracket_{\mathcal{J}} \subseteq \mathcal{C R}$ and $\mathrm{e} \mathcal{R}_{0} \subseteq \llbracket C \rrbracket_{\mathcal{J}} \subseteq \mathcal{C R}$. So $\mathcal{C} \mathcal{R}_{0} \subseteq\left(\llbracket \mathrm{B} \rrbracket_{\mathcal{J}} \cap \llbracket C \rrbracket_{\mathcal{J}}\right) \subseteq \mathcal{C R}$.

(3) If $\mathrm{A}=\mathrm{B} \rightarrow \mathrm{C}$ then evidently $\left(\llbracket \mathrm{B} \rrbracket_{\mathcal{J}} \rightarrow \llbracket \mathrm{C} \rrbracket_{\mathcal{J}}\right) \cap \mathcal{C R} \subseteq \mathrm{C} \mathcal{R}$. By IH, $\mathrm{e} \mathcal{R}_{0} \subseteq$ $\llbracket C \rrbracket_{\mathcal{J}}, \llbracket \mathrm{B} \rrbracket_{\mathcal{J}} \subseteq \mathcal{C} \mathcal{R}$, so $\left(\mathcal{C R} \rightarrow \mathcal{C} \mathcal{R}_{0}\right) \subseteq\left(\llbracket \mathrm{B} \rrbracket_{\mathcal{J}} \rightarrow \llbracket C \rrbracket_{\mathcal{J}}\right)$ and by Lemma $\mathrm{I3}$, $\mathcal{e} \mathcal{R}_{0} \subseteq\left(\llbracket \mathrm{B} \rrbracket_{\mathcal{J}} \rightarrow \llbracket C \rrbracket_{\mathcal{J}}\right)$. By Lemma $\mathbf{1 2}, \quad \mathcal{e} \mathcal{R}_{0} \subseteq\left(\llbracket \mathrm{B} \rrbracket_{\mathcal{J}} \rightarrow \llbracket C \rrbracket_{\mathcal{J}}\right) \cap \mathcal{C R}$.

THEOREM 15 (church-rosser for typed terms) If $\mathrm{t}$ is typed in the system $\mathrm{D}$ then $\mathrm{t}$ has $C R$.

Proof: Suppose that $x_{1}: A_{1}, \ldots, x_{k}: A_{k} \vdash t: A$ is a typing of $t$. Let $\mathcal{J}$ be an interpretation such that $\llbracket X \rrbracket_{\mathcal{J}}=\mathcal{C} \mathcal{R}$ for every type variable $X$. Then by Lemma I4 $\mathcal{C} \mathcal{R}_{0} \subseteq \llbracket A_{i} \rrbracket_{\mathcal{J}}$ for all $i \in\{1, \ldots, k\}$ and because all $x_{i}$ 's belong to $\mathcal{C} \mathcal{R}_{0}$ we have $x_{i} \in \llbracket A_{i} \rrbracket_{\mathcal{J}}$ for all $i$ 's. By the soundness theorem $t\left[x_{1} / x_{1}, \ldots, x_{k} / x_{k}\right] \in$ $\llbracket A \rrbracket_{\mathcal{J}}$, i.e. $t \in \llbracket A \rrbracket_{\mathcal{J}}$ and again by Lemma $\mathbf{I 4}, t \in \mathcal{C} \mathcal{R}$. 


\section{CONFLUENCE OF DEVELOPMENTS}

We have proved that every term $t$ typed in the system $D$ has the Church-Rosser property. Therefore the $\beta$-reduction relation for typed terms is confluent.

We proceed to prove the confluence of a "restricted" kind of reduction on the untyped terms by defining an embedding of the untyped terms into the typed terms. This "restricted" reduction defines the notion of a development [2, I3]. In our proof we are motivated by the proof of the theorem of finite developments as presented in [13, pages $\left.45^{-49}\right]$. The rest of this section up to Lemma 33 makes explicit the machinery used there.

First we need to define an operator $\left.\Psi_{(-},,_{-}\right)$such that for any pair $(t, \mathcal{F})$ with $t \in \Lambda$ and $\mathcal{F}$ a set of occurrences of redexes in $t, \Psi(t, \mathcal{F})$ will be produced from the term $t$ where all the redexes $(\lambda x . u) v$ in $t$ not belonging to $\mathcal{F}$ are "frozen" by replacing them with $((\mathrm{c}) \lambda x . u) v$, where $\mathrm{c}$ is a new distinguished variable for $\lambda$-terms that is never substituted. By doing this we leave as redexes in $t$ only the ones in $\mathcal{F}$. In addition, we will block the possibility of creating new redexes from $\beta$-reductions in $t$ out of the contraction of the redexes in $\mathcal{F}$. For example, if $t$ contains a subterm $(y) \nu$, then after $\beta$-reducing a redex in $t$, some subterm of the form $\lambda x$.u may substitute $y$ and create a new redex. In order to avoid this situation we will also put in front of every subterm of the form $(w) v$, with $w$ not a $\lambda$-abstraction, the distinguished variable $c$, i.e. we replace $(w) v$ with $((\mathrm{c}) w) v$. Thus we also "freeze" the applications in $t$ so that they cannot be transformed into redexes.

REMARK I6 In what follows, $\mathcal{F}$ is a set of occurrences of redexes in $t$, i.e. of redexes accompanied with a pointer showing their location in term $t$. For example, the same redex $(\lambda x . x) x$ occurs in two different locations in $\mathrm{t}=((\lambda x . x) x)(\lambda x . x) x$ and thus may appear twice in $\mathcal{F}$ but with a different pointer in each case.

However, for brevity reasons, we will refer to $\mathcal{F}$ as a set of redexes in $t$ and will not specify the accompanying pointer of the redexes.

DEFinition 17 Let $t \in \Lambda$ and $\mathcal{F}$ a set of redexes in $t$. We define formally the operator $\Psi\left({ }_{-},,_{-}\right)$by induction on $t$ :

(I) if $t$ is a variable $x$ then $\mathcal{F}=\emptyset$ and

$$
\Psi(x, \emptyset) \stackrel{\text { def }}{=} x
$$

(2) if $t$ is a $\lambda$-abstraction $\lambda x$.u then $\mathcal{F}$ is a set of redexes in $u$ and

$$
\Psi(\lambda x . u, \mathcal{F}) \stackrel{\text { def }}{=} \lambda x . \Psi(u, \mathcal{F})
$$

(3) if $t$ is an application $u v$ and $\mathcal{F}_{1}$ (resp. $\mathcal{F}_{2}$ ) is the set of redexes of $u$ (resp. $v)$ in $\mathcal{F}$ then $\mathcal{F} \backslash\{t\}=\mathcal{F}_{1} \cup \mathcal{F}_{2}$ and

$$
\Psi(u v, \mathcal{F}) \stackrel{\text { def }}{=} \begin{cases}(\mathfrak{c}) \Psi\left(\mathfrak{u}, \mathcal{F}_{1}\right) \Psi\left(v, \mathcal{F}_{2}\right) & \text { if } \mathrm{t} \notin \mathcal{F} \\ \Psi\left(\mathfrak{u}, \mathcal{F}_{1}\right) \Psi\left(v, \mathcal{F}_{2}\right) & \text { otherwise }\end{cases}
$$


We call $\Psi(t, \mathcal{F})$ the freezing of $(t, \mathcal{F})$.

EXAMPLE I8

I. If $t=(\lambda x . x)(\lambda x . x) y$ and $\mathcal{F}=\{(\lambda x \cdot x) y\}$, then $\Psi(t, \mathcal{F})=((c) \lambda x \cdot x)(\lambda x \cdot x) y$.

2. If $t=(\lambda x .(x) x) \lambda x .(x) x$ and $\mathcal{F}=\{(\lambda x .(x) x) \lambda x .(x) x\}$, then $\Psi(t, \mathcal{F})=(\lambda x .(c) x x) \lambda x .(c) x x$.

Let $\mathrm{c}$ be the new variable introduced above.

DEFINITION I9 We define inductively a subset of the $\lambda$-terms with $\mathrm{c}$, denoted $\Lambda_{c}$, in the following way:

(I) if $x$ is a variable distinct from $c$, then $x \in \Lambda_{c}$ (variable)

(2) if $x$ is a variable distinct from $c$ and $T \in \Lambda_{c}$, then $\lambda x . T \in \Lambda_{c}$ $(\lambda$-abstraction)

(3) if $\mathrm{T}, \mathrm{U} \in \Lambda_{\mathrm{c}}$, then (c) $\mathrm{TU} \in \Lambda_{\mathrm{c}}$ (non-redex application)

(4) if $\mathrm{T}, \mathrm{U} \in \Lambda_{\mathrm{c}}$ and $\mathrm{T}$ is a $\lambda$-abstraction, then $\mathrm{TU} \in \Lambda_{\mathrm{c}}$ (redex application)

Note that there are terms of $\Lambda$ not in $\Lambda_{c}$, for example $c,(\lambda x . x) y z$, ((c) $\lambda x . x) y z \notin \Lambda_{c}$ but $((c)(\lambda x . x) y) z,((c)((c) \lambda x . x) y) z \in \Lambda_{c}$.

LEMMA 20 (I) If $\mathrm{T}, \mathrm{U} \in \Lambda_{\mathrm{c}}$ and $\mathrm{x} \neq \mathrm{c}$, then $\mathrm{T}[\mathrm{U} / \mathrm{x}] \in \Lambda_{\mathrm{c}}$. (2) $\Lambda_{\mathrm{c}}$ is closed under $\beta$-reduction, i.e. if $\mathrm{T} \in \Lambda_{\mathrm{c}}$ and $\mathrm{T} \stackrel{*}{\longrightarrow} \beta \mathrm{T}^{\prime}$ then $\mathrm{T}^{\prime} \in \Lambda_{\mathrm{c}}$.

Proof: (I) By induction on T. (2) By induction on T using (I).

LEMMA $2 \mathrm{I}$ Every term of $\Lambda_{\mathrm{c}}$ is typed in the system $D$.

Proof: We can actually prove that for every term $T \in \Lambda_{\mathrm{c}}$ and every context $\Gamma$ for all the free variables of $\mathrm{T}$, except $\mathrm{c}$, there exist types $\mathrm{C}, \mathrm{A}$ such that $\Gamma, \mathrm{c}: \mathrm{C} \vdash_{\mathrm{D}}$ $T: A$. The proof can be found in [13, pages 46-47]. The use of intersection types is crucial in this proof but we will see later [Proposition 36 , that with a slight modification of $\Lambda_{c}$ the proof can also be adapted for the simply typed $\lambda$-calculus.

LEMMA 22 The range of the freezing operator $\left.\Psi_{(-,}\right)$is a subset of $\Lambda_{c}$.

Proof: We prove by an easy induction on $t$ that if $t \in \Lambda$ and $\mathcal{F}$ is a set of redexes in $t$, then $\Psi(t, \mathcal{F}) \in \Lambda_{c}$.

DEFINITION 23 We define a surjective mapping from $\Lambda_{c}$ onto $\Lambda$ called erasure and denoted $\left.\right|_{-} \mid$, by induction on $T \in \Lambda_{c}$ :

(I) if $\mathrm{T}$ is a variable distinct from $\mathrm{c}$, then $|\mathrm{T}| \stackrel{\text { def }}{=} \mathrm{T}$;

(2) if $T=\lambda x . U$ and $U \in \Lambda_{c}$, then $|T| \stackrel{\text { def }}{=} \lambda x .|U|$; 
(3) if $\mathrm{T}=(\mathrm{c}) \mathrm{UV}$ and $\mathrm{U}, \mathrm{V} \in \Lambda_{\mathrm{c}}$, then $|\mathrm{T}| \stackrel{\text { def }}{=}(|\mathrm{U}|)|\mathrm{V}|$;

(4) if $\mathrm{T}=(\lambda x . \mathrm{U}) \mathrm{V}$ and $\mathrm{U}, \mathrm{V} \in \Lambda_{\mathrm{c}}$, then $|\mathrm{T}| \stackrel{\text { def }}{=}(\lambda x .|\mathrm{U}|)|\mathrm{V}|$.

Thus $|\mathrm{T}|$ is obtained by leaving out the variable $\mathrm{c}$ in $\mathrm{T}$. It is noticeable that erasure does not preserve types.

We will now show, in the following four lemmas, that $\Psi\left({ }_{-},-\right)$defines a one-to-one correspondence between the pairs $(t, \mathcal{F})$ and the terms of $\Lambda_{c}$, i.e. an embedding of the untyped terms into the typed terms.

LEMMA 24 If $\mathrm{t} \in \Lambda$ and $\mathcal{F}$ is a set of redexes in $\mathrm{t}$, then $|\Psi(\mathrm{t}, \mathcal{F})|=\mathrm{t}$.

Proof: By an easy induction on $t \in \Lambda$ using Lemma 22 .

LEMMA 25 If $\mathrm{t} \in \Lambda$ and $\mathcal{F}$ is a set of redexes in $\mathrm{t}$, then $\mathcal{F}=\{|\mathrm{R}|: \mathrm{R}$ is a redex in $\Psi(\mathrm{t}, \mathcal{F})\}$.

Proof: By induction on $t \in \Lambda$ using Lemma 24

LEMMA 26 If $\mathrm{T} \in \Lambda_{\mathrm{c}}, \mathrm{t}=|\mathrm{T}|$ and $\mathcal{F}=\{|\mathrm{R}|: \mathrm{R}$ redex in $\mathrm{T}\}$ then $\mathcal{F}$ is a set (possibly empty) of redexes in $\mathrm{t}$ and $\Psi(\mathrm{t}, \mathcal{F})=\mathrm{T}$, i.e. $\Psi\left({ }_{-},,_{-}\right)$is surjective.

Proof: By induction on $\mathrm{T} \in \Lambda_{\mathrm{c}}$.

LEMMA 27 For every $\mathrm{T} \in \Lambda_{\mathrm{c}}$ there exists one and only one pair $(\mathrm{t}, \mathcal{F})$ with $\mathrm{t} \in \Lambda$ and $\mathcal{F}$ a set of redexes in $\mathrm{t}$, such that $\Psi(\mathrm{t}, \mathcal{F})=\mathrm{T}$. Therefore $\Psi\left({ }_{-},-\right)$is a one-to-one mapping onto $\Lambda_{\mathrm{c}}$.

Proof: Due to the previous lemma it suffices to prove the "only one" part. This is easily proved using Lemma 24 and Lemma 25.

DEFINITION 28 Let $t \in \Lambda, \mathcal{F}$ a set of redexes in $t$ and $t \longrightarrow \beta t_{1}$ by contraction of a redex $r$ in $t$. If $T=\Psi(t, \mathcal{F} \cup\{r\}), R$ the redex in $T$ with $|R|=r[$ Lemma 25] and $\mathrm{T}_{1}$ the term obtained by contraction of $\mathrm{R}$ in $\mathrm{T}$, then by Lemma 27 there exists $\mathcal{F}_{1}$ such that $\Psi\left(t_{1}, \mathcal{F}_{1}\right)=T_{1}$ (in fact $t_{1}=\left|T_{1}\right|$ and $\mathcal{F}_{1}=\left\{|R|: R\right.$ redex in $\left.T_{1}\right\}$ ). We call $\mathcal{F}_{1}$ the set of residuals of $\mathcal{F}$ in $\mathrm{t}_{1}$ relative to $\mathrm{r}$.

EXAMPLE 29 Let $t=(\lambda x .(x) x) \lambda x .(x) x \longrightarrow \beta(\lambda x .(x) x) \lambda x .(x) x=t_{1}$ and $\mathcal{F}=$ $\{(\lambda x .(x) x) \lambda x .(x) x\}$. Then

$$
\begin{aligned}
\mathrm{T} & =\Psi(\mathrm{t}, \mathcal{F})=(\lambda x \cdot(\mathrm{c}) x x) \lambda x \cdot(\mathrm{c}) \times x \\
\mathrm{~T}_{1} & =((\mathrm{c}) \lambda x \cdot(\mathrm{c}) \times x) \lambda x .(\mathrm{c}) \times x \\
\mathcal{F}_{1} & =\emptyset
\end{aligned}
$$

So the set of residuals of $\mathcal{F}$ in $t_{1}$ relative to redex $(\lambda x .(x) x) \lambda x .(x) x$ is $\emptyset$.

EXAMPLE 30 Let $t=(\lambda x .(x) x)(\lambda x . x) x \longrightarrow_{\beta}((\lambda x . x) x)(\lambda x . x) x=t_{1}$ and $\mathcal{F}=$ $\{(\lambda x \cdot x) x\}$. Then

$$
\begin{aligned}
\mathrm{T} & =\Psi(\mathrm{t}, \mathcal{F} \cup\{(\lambda x \cdot(x) x)(\lambda x \cdot x) x\})=(\lambda x \cdot(\mathrm{c}) x x)(\lambda x \cdot x) x \\
\mathrm{~T}_{1} & =((\mathrm{c})(\lambda x \cdot x) x)(\lambda x \cdot x) x \\
\mathcal{F}_{1} & =\{(\lambda x \cdot x) x,(\lambda x \cdot x) x\}
\end{aligned}
$$


So the set of residuals of $\mathcal{F}$ in $t_{1}$ relative to redex $(\lambda x .(x) x)(\lambda x . x) x$ is $\{(\lambda x . x) x$, $(\lambda x . x) x\}$, i.e. two distinct occurrences of the same redex $(\lambda x . x) x$.

Definition 3I Let $t \in \Lambda, \mathcal{F}$ a set of redexes in $t$ and the $\beta$-reduction

$$
t \longrightarrow \beta t_{1} \longrightarrow_{\beta} \ldots t_{n-1} \longrightarrow_{\beta} t_{n}
$$

obtained by contracting consecutively the redexes $r$ in $t, r_{1}$ in $t_{1}, \ldots, r_{n-1}$ in $t_{n-1}$. We define, by induction on $n$, the set $\mathcal{F}_{n}$ of residuals of $\mathcal{F}$ in $t_{n}$ relative to $\left(r, r_{1}, \ldots, r_{n-1}\right):$ if $n=1$ then $\mathcal{F}_{1}$ is defined above; if $n \geqslant 2$ then $\mathcal{F}_{n}$ is the set of residuals of $\mathcal{F}_{n-1}$ in $t_{n}$ relative to $r_{n-1}$ where $\mathcal{F}_{n-1}$ is the set of residuals of $\mathcal{F}$ in $t_{n-1}$ relative to $\left(r, r_{1}, \ldots, r_{n-2}\right)$.

Intuitively, given a $\beta$-reduction of a term $t$ we select a set $\mathcal{F}$ of redexes in the term, we "mark" those redexes (in $\Lambda_{c}$ we "freeze" all the other redexes by blocking them with the variable c) and we follow their evolution throughout the reduction.

Definition 32 Let $t \in \Lambda$ and $\mathcal{F}$ a set of redexes in $t$. A development of $(t, \mathcal{F})$ is a $\beta$-reduction $t \longrightarrow \beta t_{1} \longrightarrow \beta \quad \ldots t_{n-1} \longrightarrow \beta t_{n} \quad(n \geqslant 0)$ obtained by contracting consecutively the redexes $r, r_{1}, \ldots, r_{n-1}$ where $r \in \mathcal{F}$ and $r_{i}$ is a residual of $\mathcal{F}$ in $t_{i}$ relative to $\left(r, r_{1}, \ldots, r_{i-1}\right)$, for all $i$. If $\mathcal{F}_{n}$ is the set of residuals of $\mathcal{F}$ in $t_{n}$ relative to $\left(r, r_{1}, \ldots, r_{n-1}\right)$ then we denote the development by $(t, \mathcal{F}) \stackrel{*}{\longrightarrow} d\left(t_{n}, \mathcal{F}_{n}\right)$ or $t \stackrel{\mathcal{F}}{\longrightarrow} t_{n}$. As in the case of $\beta$-reduction we write $(t, \mathcal{F}) \longrightarrow_{d}\left(t_{1}, \mathcal{F}_{1}\right)$ for the one-step development where $t \longrightarrow \beta t_{1}$.

In a development of $(t, \mathcal{F})$ we always contract redexes that are residuals of the initial set $\mathcal{F}$ of redexes. This is achieved within $\Lambda_{c}$ by "freezing" the applications in $t$, blocking them with the variable $c$, so that they will not become redexes themselves.

LEMMA 33 Let $\mathrm{t} \in \Lambda$ and $\mathcal{F}$ a set of redexes in $\mathrm{t}$. There exists a one-to-one correspondence between the developments of $(\mathrm{t}, \mathcal{F})$ and the $\beta$-reductions of $\Psi(t, \mathcal{F})$.

Proof: It suffices to show that

$$
(\mathrm{t}, \mathcal{F}) \longrightarrow_{\mathrm{d}}\left(\mathrm{t}^{\prime}, \mathcal{F}^{\prime}\right) \Leftrightarrow \Psi(\mathrm{t}, \mathcal{F}) \longrightarrow_{\beta} \Psi\left(\mathrm{t}^{\prime}, \mathcal{F}^{\prime}\right)
$$

But this is immediate from Lemma 27 and the way of defining residuals.

THEOREM 34 (confluence of developments) If $\mathrm{t} \stackrel{\mathcal{F}_{1}}{\longrightarrow} \mathrm{d}_{\mathrm{t}}$ and $\mathrm{t} \stackrel{\mathcal{F}_{2}}{\longrightarrow} \mathrm{d}_{\mathrm{d}} \mathrm{t}_{2}$ then there exist sets $\mathcal{F}^{1}$, resp. $\mathcal{F}^{2}$, of redexes in $\mathrm{t}_{1}$, resp. $\mathrm{t}_{2}$, and a term $\mathrm{t}_{3} \in \Lambda$ such that $\mathrm{t}_{1} \stackrel{\mathcal{F}^{1}}{\longrightarrow} \mathrm{t}_{3}$ and $\mathrm{t}_{2} \stackrel{\mathcal{F}^{2}}{\longrightarrow} \mathrm{t}_{3}$.

Proof: The proof is sketched in Figure 2. Let $t \stackrel{\mathcal{F}_{1}}{\longrightarrow} t_{1}$ and $t \stackrel{\mathcal{F}_{2}}{\longrightarrow} t_{2}$. Then there exist $\mathcal{F}_{1}^{1}, \mathcal{F}_{2}^{2}$ such that $\left(t, \mathcal{F}_{1}\right) \stackrel{*}{\longrightarrow} \mathrm{d}\left(t_{1}, \mathcal{F}_{1}^{1}\right)$ and $\left(t, \mathcal{F}_{2}\right) \stackrel{*}{\longrightarrow} \mathrm{d}\left(t_{2}, \mathcal{F}_{2}^{2}\right)$. By extending the initial sets of redexes $\mathcal{F}_{1}, \mathcal{F}_{2}$ to $\mathcal{F}_{1} \cup \mathcal{F}_{2}$ and contracting the same redexes, we get the developments $\left(t, \mathcal{F}_{1} \cup \mathcal{F}_{2}\right) \stackrel{*^{*}}{\longrightarrow}\left(t_{1}, \mathcal{F}_{1}^{1} \cup \mathcal{F}_{2}^{1}\right)$ and $\left(t, \mathcal{F}_{1} \cup\right.$ $\left.\mathcal{F}_{2}\right) \stackrel{*}{\longrightarrow}\left(t_{2}, \mathcal{F}_{1}^{2} \cup \mathcal{F}_{2}^{2}\right)$ for some $\mathcal{F}_{2}^{1}$ (resp. $\mathcal{F}_{1}^{2}$ ) which are the residuals of $\mathcal{F}_{2}$ 

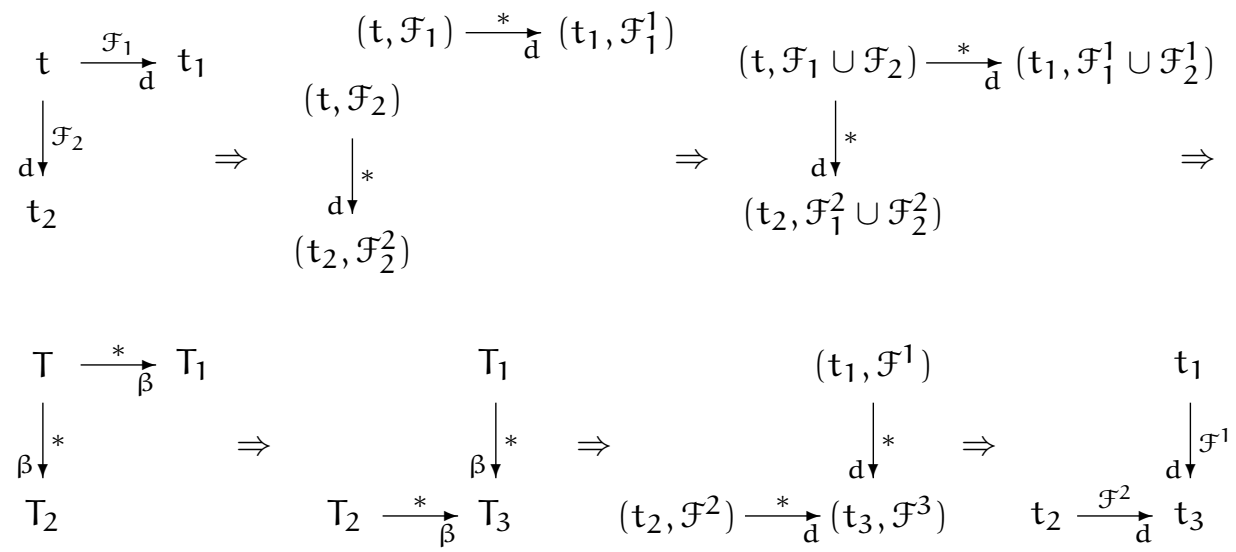

Figure 2: Sketching the proof of Theorem 34

(resp. $\mathcal{F}_{1}$ ) for the corresponding reductions from $t / 4$ Let $\mathcal{F}^{1}=\mathcal{F}_{1}^{1} \cup \mathcal{F}_{2}^{1}$ and $\mathcal{F}^{2}=\mathcal{F}_{1}^{2} \cup \mathcal{F}_{2}^{2}$. By Lemma 33 there exist $T, T_{1}, T_{2} \in \Lambda_{c}$ such that

$$
\mathrm{T}=\Psi\left(\mathrm{t}, \mathcal{F}_{1} \cup \mathcal{F}_{2}\right), \quad \mathrm{T}_{1}=\Psi\left(\mathrm{t}_{1}, \mathcal{F}^{1}\right), \quad \mathrm{T}_{2}=\Psi\left(\mathrm{t}_{2}, \mathcal{F}^{2}\right)
$$

and $\mathrm{T} \stackrel{*}{\longrightarrow} \beta \mathrm{T}_{1}, \mathrm{~T} \stackrel{*}{\longrightarrow} \mathrm{T}_{2}$. But $\mathrm{T}$ is typed in the system $D$ [Lemma $2 \mathrm{I}$ ] therefore $T$ has CR [Theorem ${ }_{15}$. So there exist $T_{3} \in \Lambda_{c}$ such that $T_{1} \rightarrow_{\beta} T_{3}$, $\mathrm{T}_{2} \stackrel{*}{\longrightarrow} \beta \mathrm{T}_{3}$. By Lemma 33 there exist $t_{3}, \mathcal{F}^{3}$ such that $\mathrm{T}_{3}=\Psi\left(\mathrm{t}_{3}, \mathcal{F}^{3}\right)$ and

$$
\left(\mathrm{t}_{1}, \mathcal{F}^{1}\right) \stackrel{*}{\longrightarrow} \mathrm{d}\left(\mathrm{t}_{3}, \mathcal{F}^{3}\right), \quad\left(\mathrm{t}_{2}, \mathcal{F}^{2}\right) \stackrel{*}{\longrightarrow} \mathrm{d}\left(\mathrm{t}_{3}, \mathcal{F}^{3}\right)
$$

i.e. $t_{1} \stackrel{\mathcal{F}^{1}}{\longrightarrow} t_{3}$ and $t_{2} \stackrel{\mathcal{F}^{2}}{\longrightarrow} t_{3}$.

\section{I DIGRESSION: A PROOF WITHOUT INTERSECTION TYPES}

In the above proof we made use of the fact that typability in $\mathrm{D}$ implies the Church-Rosser property [Theorem I5] and for this reason we employed the "freezing" mechanism of $\Lambda_{c}$ to simulate the process of a development. We can however prove Theorem 34 without introducing system $\mathrm{D}$ [I2]. All we need is the simply typed $\lambda$-calculus $\lambda \rightarrow$ for which the analogous Church-Rosser theorem, i.e. typability in $\lambda_{\rightarrow}$ implies the Church-Rosser property, can be found in [IO, [5].

The simply typed $\lambda$-calculus can be defined as a restriction of system $D$ by ommitting the intersection types and the corresponding rules ( $\cap_{1}$-elimination), $\left(\cap_{2}\right.$-elimination), and ( $\cap$-introduction). The typing relation will be denoted by $\vdash_{\lambda_{\rightarrow}}$.

The "freezing" mechanism of $\Lambda_{c}$ must now be adapted to the new situation. We consider a denumerable set $\mathcal{C}=\left\{\mathrm{c}_{0}, \mathrm{c}_{1}, \ldots\right\}$ of new distinguished variables.

${ }^{4}$ The residuals of a set of redexes are determined by the residuals of the individual redexes. 
Definition 35 We define inductively a subset of the $\lambda$-terms with the variables $c_{0}, c_{1}, \ldots$, denoted $\Lambda_{\overline{\mathbf{c}}}$, as before:

(I) if $x$ is a variable distinct from $c_{0}, c_{1}, \ldots$, then $x \in \Lambda_{\overline{\mathbf{c}}}$ (variable)

(2) if $x$ is a variable distinct from $c_{0}, c_{1}, \ldots$ and $T \in \Lambda_{\overline{\mathbf{c}}}$, then $\lambda x . T \in \Lambda_{\overline{\mathbf{c}}}$ $(\lambda$-abstraction)

(3) if $\mathrm{T}, \mathrm{U} \in \Lambda_{\overline{\mathbf{c}}}$, then $\left(\mathrm{c}_{i}\right) \mathrm{TU} \in \Lambda_{\overline{\mathbf{c}}}$ for any $i$ (non-redex application)

(4) if $T, U \in \Lambda_{\overline{\mathbf{c}}}$ and $\mathrm{T}$ is a $\lambda$-abstraction, then $\mathrm{TU} \in \Lambda_{\overline{\mathbf{c}}}$ (redex application)

Any term of $\Lambda_{\overline{\mathbf{c}}}$ can be transformed to a term of $\Lambda_{\mathrm{c}}$ by just replacing the variables $c_{0}, c_{1}, \ldots$ with $c$. We can easily prove as before that the set $\Lambda_{\overline{\mathbf{c}}}$ is closed under $\beta$-reduction and that every term of $\Lambda_{\overline{\mathbf{c}}}$ is typed in the system D.

PROPOSITION 36 Let $\mathrm{T} \in \Lambda_{\overline{\mathbf{c}}}$ be a term where each of the variables $\mathrm{c}_{0}, \mathrm{c}_{1}, \ldots$ has at most one occurrence, and $\Gamma$ be any context for the free variables of $\mathrm{T}$, except $\mathrm{c}_{0}, \mathrm{c}_{1}, \ldots$. Then there exist types $A, C_{0}, C_{1}, \ldots, C_{n}$ of the simply-typed $\lambda$-calculus such that $\Gamma, c_{0}$ : $\mathrm{C}_{0}, \ldots, \mathrm{c}_{\mathrm{n}}: \mathrm{C}_{\mathrm{n}} \vdash_{\lambda \rightarrow} \mathrm{T}: \mathrm{A}$.

Proof: We use induction on T. We sketch the proof for the last two cases (3) and (4).

(3) Let $T=c_{0} U V$. From hypothesis we can suppose that $F V(U) \cap \mathcal{C}=$ $\left\{c_{1}, \ldots, c_{k}\right\}$ and $F V(V) \cap \mathcal{C}=\left\{c_{k+1}, \ldots, c_{n}\right\}$. By IH, there exist types $B, D$, $\mathrm{C}_{1}, \ldots, \mathrm{C}_{\mathrm{k}}, \mathrm{C}_{\mathrm{k}+1}, \ldots \mathrm{C}_{\mathrm{n}}$ such that

$$
\Gamma, c_{1}: C_{1}, \ldots, c_{k}: C_{k} \vdash_{\lambda_{\rightarrow}} U: B
$$

and

$$
\Gamma, c_{k+1}: C_{k+1}, \ldots, c_{n}: C_{n} \vdash_{\lambda \rightarrow} V: D
$$

Then we can deduce that

$$
\Gamma, c_{1}: C_{1}, \ldots, c_{k}: C_{k}, c_{k+1}: C_{k+1}, \ldots, c_{n}: C_{n}, c_{0}: B \rightarrow D \rightarrow A \vdash_{\lambda_{\rightarrow}} c_{0} U V: A
$$

(4) Let $\mathrm{T}=(\lambda x . \mathrm{V}) \mathrm{U}$. From hypothesis we can suppose that $\mathrm{FV}(\mathrm{U}) \cap$ $\mathcal{C}=\left\{\mathrm{c}_{0}, \ldots, \mathrm{c}_{\mathrm{k}}\right\}$ and $\mathrm{FV}(\mathrm{V}) \cap \mathcal{C}=\left\{\mathrm{c}_{\mathrm{k}+1}, \ldots, \mathrm{c}_{\mathrm{n}}\right\}$. By IH, there exist types $\mathrm{B}, \mathrm{C}_{0}, \ldots, \mathrm{C}_{\mathrm{k}}$ such that

$$
\Gamma, c_{0}: C_{0}, \ldots, c_{k}: C_{k} \vdash_{\lambda \rightarrow} U: B
$$

By IH again, for the context $\Gamma, x: B$ there exist types $A, C_{k+1}, \ldots, C_{n}$ such that

$$
\Gamma, x: B, c_{k+1}: C_{k+1}, \ldots, c_{n}: C_{n} \vdash_{\lambda_{\rightarrow}} V: A
$$

Then we can easily deduce

$$
\Gamma, c_{0}: C_{0}, \ldots, c_{k}: C_{k}, c_{k+1}: C_{k+1}, \ldots, c_{n}: C_{n} \vdash_{\lambda \rightarrow}(\lambda x . V) U: A
$$


The cases (I) and (2) are immediate.

So the terms of $\Lambda_{\overline{\mathbf{c}}}$ with at most one occurrence of $c_{0}, c_{1}, \ldots$ are typable in the simply-typed $\lambda$-calculus and as we stated above they have the ChurchRosser property. Consider now the terms $T, T_{1}, T_{2}$ in the proof of Theorem 34 . If we replace each occurrence of the variable $c$ in $T$ by a new variable in $\mathcal{C}$ we get a term $T^{\prime} \in \Lambda_{\overline{\mathbf{c}}}$ with at most one occurrence of $c_{0}, c_{1}, \ldots$ and with the same redexes as $T$. By simulating the reductions $T \stackrel{*}{\longrightarrow} \beta T_{1}$ and $T \stackrel{*}{\longrightarrow} \beta T_{2}$ we get in $\Lambda_{\overline{\mathbf{c}}}$ the reductions $T^{\prime} \stackrel{*}{\longrightarrow}_{\beta} T_{1}^{\prime}$ and $T^{\prime} \stackrel{*}{\longrightarrow}_{\beta} T_{2}^{\prime}$ where $T_{1}^{\prime}, T_{2}^{\prime}$ have the same redexes as $T_{1}, T_{2}$, respectively. Because $T^{\prime}$ has $C R$, there exists a term $T_{3}^{\prime} \in \Lambda_{\overline{\mathbf{c}}}$ such that $\mathrm{T}_{1}^{\prime} \stackrel{*}{\longrightarrow}_{\beta} \mathrm{T}_{3}^{\prime}$ and $\mathrm{T}_{2}^{\prime} \stackrel{*}{\longrightarrow} \beta \mathrm{T}_{3}^{\prime}$. It remains to replace the variables $c_{0}, c_{1}, \ldots$ by $\mathrm{c}$ and recover the term $T_{3} \in \Lambda_{c}$ and the reductions $T_{1} \stackrel{*}{\longrightarrow} \beta T_{3}, T_{2} \stackrel{*}{\longrightarrow} \beta T_{3}$. Then we can proceed with the rest of the proof of Theorem 34 .

\section{APPLICATION: THE CHURCH-ROSSER THEOREM}

DEFINITION 37 We define a reduction relation on $\Lambda$ called one-reduction (notation $\stackrel{*}{\longrightarrow}$ ) by: $\mathrm{t} \stackrel{*}{\longrightarrow} 1 \mathrm{t}^{\prime} \stackrel{\text { def }}{\Longleftrightarrow} \exists \mathcal{F}, \mathcal{F}^{\prime}$ such that $(\mathrm{t}, \mathcal{F}) \stackrel{*}{\longrightarrow} \mathrm{d}\left(\mathrm{t}^{\prime}, \mathcal{F}^{\prime}\right)$.

LEMMA $38 \stackrel{*}{\longrightarrow}$ is the transitive closure of $\stackrel{*}{\longrightarrow} 1$.

Proof: Let $t \stackrel{*}{\longrightarrow} \beta t^{\prime}$. We use induction on the length $n$ of the reduction. If $n=0$, i.e. $t^{\prime}=t$, then for some $\mathcal{F}, \quad(t, \mathcal{F}) \stackrel{*}{\longrightarrow}_{d}(t, \mathcal{F})$ trivially. If $n \geqslant 1$ then $t \stackrel{*}{\longrightarrow} \beta t^{\prime \prime} \longrightarrow \beta t^{\prime}$ for some $t^{\prime \prime} \in \Lambda$. By IH there exist terms $t_{1}, t_{2}, \ldots, t_{k} \in \Lambda$ such that

$$
\mathrm{t} \stackrel{*}{\longrightarrow} 1 \mathrm{t}_{1} \stackrel{*}{\longrightarrow} 1 \mathrm{t}_{2} \ldots \stackrel{*}{\longrightarrow} 1 \mathrm{t}_{\mathrm{k}} \stackrel{*}{\longrightarrow} 1 \mathrm{t}^{\prime \prime}
$$

If $r$ is the reduced redex in $t^{\prime \prime} \longrightarrow \beta t^{\prime}$ then $\left(t^{\prime \prime},\{r\}\right) \longrightarrow_{d}\left(t^{\prime}, \emptyset\right)$, so $t^{\prime \prime} \stackrel{*}{\longrightarrow} t^{\prime}$, i.e. finally

$$
\mathrm{t} \stackrel{*}{\longrightarrow} 1 \mathrm{t}_{1} \stackrel{*}{\longrightarrow} 1 \mathrm{t}_{2} \ldots \stackrel{*}{\longrightarrow} \mathrm{t}_{\mathrm{k}} \stackrel{*}{\longrightarrow} 1 \mathrm{t}^{\prime \prime} \stackrel{*}{\longrightarrow} 1 \mathrm{t}^{\prime}
$$

THEOREM 39 (church-rosser) If $\mathrm{t} \in \Lambda$ then $\mathrm{t}$ has $C R$.

Proof: We have to show that if $t \stackrel{*}{\longrightarrow} \beta t_{1}$ and $t \stackrel{*}{\longrightarrow} \beta t_{2}$ then there exists $t_{3} \in \Lambda$ such that $t_{1} \stackrel{*}{\longrightarrow} t_{3}$ and $t_{2} \stackrel{*}{\longrightarrow} t_{3}$. This is immediate from Lemma 38 and Theorem 34 by a simple diagram chasing of Figure 3

\section{ACKNOWLEDGEMENT}

The authors would like to thank the two anonymous referees for their very helpful comments.

\footnotetext{
5This relation is almost the same as the one defined in [2, Definition II.2.27] with the difference that there $\mathcal{F}^{\prime}=\emptyset$. The reason is that when $\mathcal{F}^{\prime}=\emptyset$ all residuals are "consumed" and the development ends with a unique term [2, Theorem II.2.25].
} 


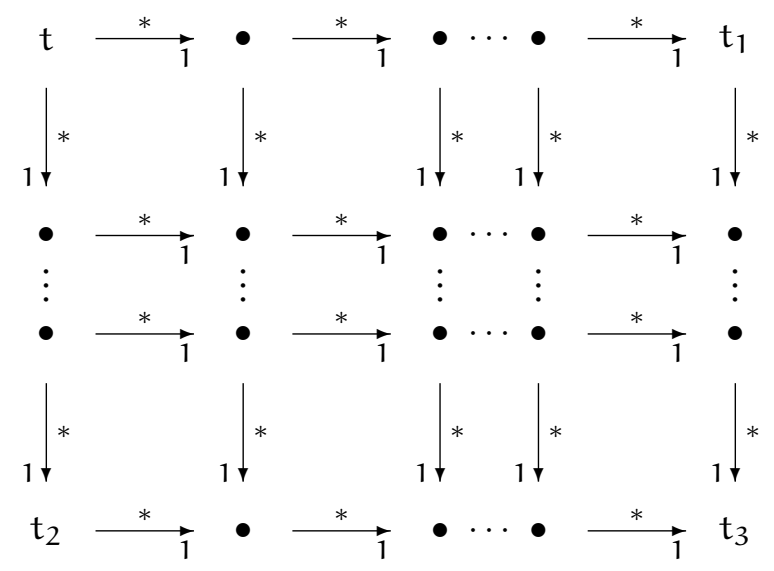

Figure 3: Diagram of reductions for the proof of Theorem 39

\section{REFERENCES}

[I] van Bakel S., Intersection Type Disciplines in Lambda Calculus and Applicative Term Rewriting Systems, PhD thesis, University of Nijmegen, 1993

[2] Barendregt H.P., The Lambda Calculus, Its Syntax and Semantics, 2nd edition, North-Holland , I984

[3] Church A. and Rosser J.B., Some properties of conversion, Trans. Amer. Math. Soc. 39, I936, pp. 472-482

[4] Coppo M. and Dezani-Ciancaglini M., A new type assignment for $\lambda$ terms, Archiv für Mathematische Logik 19, 1978, pp. 139-156

[5] Coppo M. and Dezani-Ciancaglini M., An extension of basic functionality theory for lambda-calculus, Notre Dame Fournal of Formal Logic 21, I980, pp. 685-693

[6] Coppo M., Dezani-Ciancaglini M., and Venneri B., Principal type schemes and $\lambda$-calculus semantics, in: J.R. Hindley, J.P. Seldin (eds.), To H.B. Curry: Essays on Combinatory Logic, Typed Lambda Calculus and Formalism, Academic Press, 1980, pp. 535-560

[7] Gallier J., On the Correspondence between proofs and $\lambda$-terms, in: $\mathrm{Ph}$. de Groote (ed.), The Curry-Howard isomorphism, Cahiers du Centre de Logique 8, Université catholique de Louvain, I995, pp. 55-138

[8] Gallier J., Typing untyped $\lambda$-terms, or reducibility strikes again!, Annals of Pure and Applied Logic 9I, 1998, pp. 23I-270 
[9] Ghilezan S. and Likavec S., Reducibility: a ubiquitous method in lambda calculus with intersection types, Electronic Notes in Theoretical Computer Science $7 \mathrm{O}(\mathrm{I})$, Elsevier, 2002

[Io] Koletsos G., Church-Rosser theorem for typed functional systems, fournal of Symbolic Logic 50, 1985, pp. 782-790

[II] Koletsos G. and Stavrinos G., The structure of the reducibility proofs, in: $\mathrm{Ph}$. Kolaitis, G. Koletsos (eds.), Proceedings of the 2nd Panhellenic Logic Symposium, Delphi, 1999, pp. 138-143

[12] Koletsos G. and Stavrinos G., Properties of developments via simple types, submitted, 2007

[13] Krivine J.-L., Lambda-calcul, types et modèles, Masson, 1990 (english translation: Ellis Horwood, 1993)

[14] Newman M.H.A., On theories with a combinatorial definition of "equivalence", Annals of Mathematics 43(2), 1942, pp. 223-243

[15] Statman R., Logical relations and the simply typed lambda calculus, Information and Control 65, 1985, pp. 85-97

[16] Stavrinos G., Intersection types and reduction properties in $\lambda$-calculus, Doctoral thesis (in greek), National Technical University of Athens, 20OI 
The Australasian fournal of Logic (ISSN I448-5052) disseminates articles that significantly advance the study of logic, in its mathematical, philosophical or computational guises. The scope of the journal includes all areas of logic, both pure and applied to topics in philosophy, mathematics, computation, linguistics and the other sciences.

Articles appearing in the journal have been carefully and critically refereed under the responsibility of members of the Editorial Board. Only papers judged to be both significant and excellent are accepted for publication.

The journal is freely available at the journal website at

http://www.philosophy.unimelb.edu.au/ajl/

All issues of the journal are archived electronically at the journal website.

SubSCRIPTIONS Individuals may subscribe to the journal by sending an email, including a full name, an institutional affiliation and an email address to the managing editor at ajl-editors@unimelb.edu.au Subscribers will receive email abstracts of accepted papers to an address of their choice. For institutional subscription, please email the managing editor at ajl-editors@unimelb.edu.au

Complete published papers may be downloaded at the journal's website at http: //www.philosophy.unimelb.edu.au/ajl/ The journal currently publishes in pdf format.

Submission The journal accepts submissions of papers electronically. To submit an article for publication, send the $\mathrm{IT}_{\mathrm{E}} \mathrm{X}$ source of a submission to a member of the editorial board. For a current list of the editorial board, consult the website.

The copyright of each article remains with the author or authors of that article. 